\title{
Resonant Cavity LED's Optimized for Coupling to Polymer Optical Fibers
}

Ronny Bockstaele, Student Member, IEEE, Carl Sys, Johan Blondelle, Bart Dhoedt, Ingrid Moerman, Member, IEEE, Peter Van Daele, Member, IEEE, Piet Demeester, Senior Member, IEEE, and Roel Baets, Senior Member, IEEE

\begin{abstract}
Planar resonant-cavity light-emitting diodes have been optimized to meet optical interconnect requirements. The microcavity effect is exploited to increase the extraction efficiency into a given numerical aperture and to reduce the crosstalk in parallel optical interconnect applications. Devices are fabricated with an overall quantum efficiency of $3.7 \%$ into a polymer optical fiber with a numerical aperture of 0.5 and a FWHM beam divergence angle of $105^{\circ}$ at a drive current of $1 \mathrm{~mA}$.
\end{abstract}

Index Terms - Light-emitting diodes, microcavity, polymer optical fiber, resonant cavity.

\section{INTRODUCTION}

$\mathbf{L}$ IGHT-EMITTING diodes (LED's) are key components in the optoelectronics industry. Their advantages are robustness, reliability, low temperature sensitivity, low cost and eye safety. In high-performance telecommunication systems however, due to the high-modulation bandwidth and power level requirements, laser diodes are used. New growing application areas, such as FTTH and low-cost parallel interconnects, requiring low-cost, highly reliable, and eyesafe components, offer new market segments for LED's. In guided-wave interconnect applications, light is coupled into a waveguide, e.g., based on glass fiber [with a typical numerical aperture (NA) of 0.2] or polymer optical fiber (with a typical NA of 0.5). The optical sources should be optimized such that coupled optical power is maximized, while avoiding crosstalk. This implies that the emitted light beam should exhibit low divergence, and the extraction efficiency into the numerical aperture of the waveguide is a key parameter.

The microcavity effect has been shown to influence the spontaneous emission process such that extraction efficiencies up to 10 times higher than in conventional planar LED's are obtained [1]. The microcavity effect provides a redistribution of emitted light through enhancement or inhibition of spontaneous emission, depending on the position of the emission dipole with respect to the cavity standing wave pattern. This effect is used in resonant cavity LED's (or microcavity LED's) to influence the overall quantum efficiency $(\mathrm{QE})$, the far-

Manuscript received November 9, 1998. This work was supported by the IWT, Vlaams Instituut voor de bevordering van het WetenschappelijkTechnologisch onderzoek in de industrie, by the European Community within the framework of ESPRIT-OIIC Project 22641, and by the Belgian DWTC through the IUAP-13 project.

R. Bockstaele, C. Sys, B. Dhoedt, I. Moerman, P. Van Daele, P. Demeester, and R. Baets are with the University of Gent-IMEC, INTEC Department, B-9000 Gent, Belgium.

J. Blondelle was with the University of Gent-IMEC, B-9000 Gent, Belgium. He is now with Xeikon, 2640 Mortsel, Belgium.

Publisher Item Identifier S 1041-1135(99)01116-7.

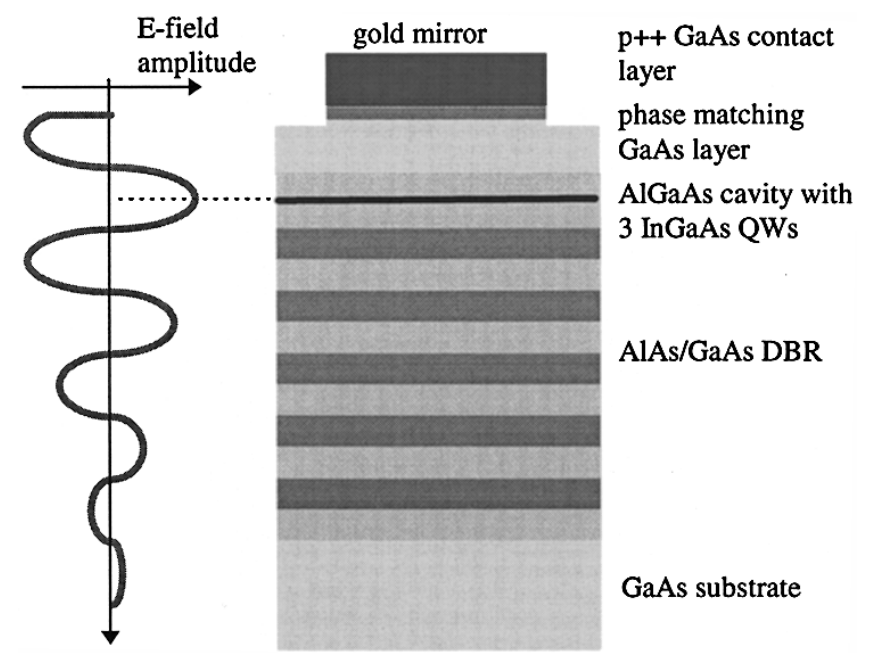

Fig. 1. Layer structure of RCLED, with standing wave pattern.

field pattern and/or the spectral width of the emitted light. Moreover, these devices show good modulation characteristics [2]. Other work has been done to integrate the resonantcavity LED (RCLED) with active drive circuits [3] and to decrease the full-width at half-maximum (FWHM) emission angle [4]. Perhaps the biggest success achieved with RCLED's has been to increase the overall QE of planar LED's to $22.3 \%$ [5] (this high efficiency is a result of a combination of the microcavity effect and photon-recycling, implying relatively large device diameters). In this letter, we present theoretical and experimental results on the optimization of small diameter RCLED's for low-beam divergence and high overall QE into a limited NA of 0.5 .

\section{Simulations}

The generic microcavity structure under investigation is shown in Fig. 1, and consists of a gold mirror, an AlGaAs $\lambda$-cavity and a GaAs-AlAs distributed Bragg reflector (DBR). An InGaAs active layer (emitting at $980 \mathrm{~nm}$ ) was chosen to study the effect of a microcavity to enhance the coupling to NA, although the absorption of the polymer optical fiber (POF) is large (about $10 \mathrm{~dB} / \mathrm{m}$ ) at these wavelengths. However, at short distance communications (e.g., parallel interconnects between chips), this attenuation becomes negligible.

Simulations were done to study the effect of the cavity length and the number of DBR pairs on the beam divergence and the extraction efficiency into the limited NA (i.e., the number of photons escaping the cavity into the NA to the number of emitted photons by the active layer). A dedicated 


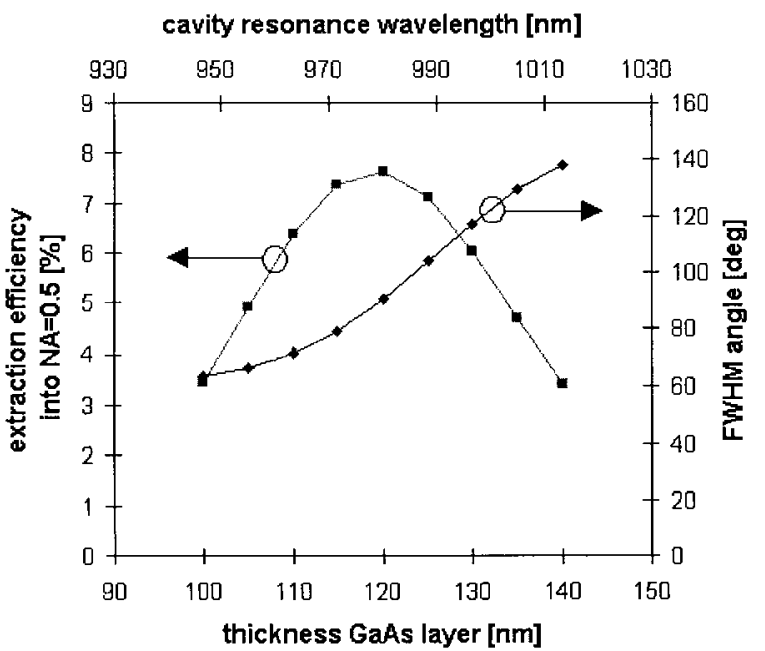

Fig. 2. Calculated extraction efficiency into NA $=0.5$ and FWHM angle of planar RCLED structure shown in Fig 1.

RCLED model was used to evaluate numerically the extraction efficiency of several structures [6]. This model takes into account radiating as well as guided modes. It was found that up to $34 \%$ of the spontaneous emitted power from the active layer is captured in laterally guided modes of the cavity. Fortunately, part of these photons can be reabsorbed by the active layers and reemitted subsequently. This "photon recycling" effect considerably increases the overall QE of the device, as an emitted photon has a higher probability to leave the cavity. It also reduces the modulation speed of the RCLED's.

The reabsorption effect decreases at higher current levels as the carriers density comes closer to the transparency carrier density. Moreover, a part of the guided photons will escape the cavity at the border of the LED before they get reabsorbed by the active layer. For high current densities and small devices (diameter smaller than the absorption length of the waveguide), these edge effects become dominant and there will be no increase of efficiency nor decrease of modulation frequency. Modulation experiments on these devices have been carried out, and error-free transmission (BER $<10^{-12}$ ) has been proven at $600 \mathrm{Mb} / \mathrm{s}$ [7].

The simulation results are shown in Fig. 2, where the extraction efficiency into NA $=0.5$ and the FWHM angle of the RCLED are plotted as a function of the thickness of the GaAs phase matching layer and corresponding cavity resonance wavelength. The total-cavity thickness can be calculated from the resonance condition: the electrical field amplitude at the active layer position should be maximized for the quantum well peak emission wavelength. This corresponds to a GaAs layer of $119.5 \mathrm{~nm}$. However, a small "detuning" further increases the extraction efficiency, as this implies an increased extraction efficiency for the nonperpendicular propagating modes. The optimal GaAs layer thickness was found to be $121 \mathrm{~nm}$, the corresponding cavity resonance wavelength is $981 \mathrm{~nm}$.

The influence of the DBR-reflectivity was also evaluated, and it was found that the optimal reflectivity of the DBR corresponds to a cavity bandwidth equal to the intrinsic spontaneous emission spectral width (i.e., without cavity). As this spectral width depends on the current density, it implies that the extraction efficiency also depends on the current density, and RCLED's driven at higher currents will exhibit a decreased extraction efficiency. The optimal reflection of the DBR at an intrinsic spontaneous emission spectral width of $30 \mathrm{~nm}$ (corresponding to a current density in the order of $100 \mathrm{~A} / \mathrm{cm}^{2}$ ), is $70.8 \%$ amplitude reflection $(50.2 \%$ power reflection), corresponding to a five pairs GaAs-AlAs DBR. Fig. 2 shows the tradeoff between a low beam divergence and a high extraction efficiency into $\mathrm{NA}=0.5$.

For the given device structure, the maximal extraction efficiency into $\mathrm{NA}=0.5$ is found to be $7.8 \%$. In that case, the total extraction efficiency is $19.7 \%$.

\section{DEVICE FABRICATION}

The layer structure as shown in Fig. 1 was grown on an n-doped GaAs substrate in an MOCVD reactor, and consists of $5 \mathrm{Si}$-doped DBR pairs (84.5-nm AlAs and 70-nm GaAs), followed by a $51.6-\mathrm{nm} \mathrm{Al}_{0.4} \mathrm{Ga}_{0.6} \mathrm{As}$ cladding layer, three 6nm-thick $\mathrm{In}_{0.2} \mathrm{Ga}_{0.8} \mathrm{As}$ quantum wells with 6-nm $\mathrm{Al}_{0.2} \mathrm{Ga}_{0.8} \mathrm{As}$ barrier layers and again a 50.6-nm $\mathrm{Al}_{0.4} \mathrm{Ga}_{0.6} \mathrm{As}$ cladding layer. Two layer structures with different GaAs phase matching layers determining the cavity resonance were grown to investigate the detuning effect: 108- and 120-nm-thick top AlGaAs layers, followed by a $\mathrm{p}^{++} \mathrm{GaAs}$ contact layer ( $\mathrm{Zn}$ doped) and a gold contact, also serving as the highly reflective mirror and defining the diameter of the RCLED. Different diameters are realized $(20,30,40,50,70$, and $85 \mu \mathrm{m})$. Mesas are defined by a shallow self-aligned wet etch, using the metal mirrors as mask pattern. The etch depth was about $130 \mathrm{~nm}$, just through the heavily $\mathrm{p}^{++}$doped GaAs contact layer and well above the active layer. This provides a current confinement mechanism.

The n-doped GaAs substrate was thinned down to $250 \mu \mathrm{m}$ to reduce the absorption. An AR coating was deposited on the back surface to reduce the Fresnel losses. To allow an easy fiber alignment, the RCLED's were flip-chip mounted onto a glass carrier using direct $\mathrm{Au}-\mathrm{Au}$ contacts and a UV-cured adhesive [8].

\section{MEASUREMENTS}

The overall $\mathrm{QE}$ values and divergence angles at $1 \mathrm{~mA}$ were found to be $7.2 \%$ and $85^{\circ}$ for the 108-nm RCLED, and $11.4 \%$ and $105^{\circ}$ for the $120-\mathrm{nm}$ RCLED. We calculated the QE into $\mathrm{NA}=0.5$ for a $50-\mu \mathrm{m}$-diameter device, from the measured far-field pattern, resulting in a value of $4.1 \%$. Fig. 3 compares the $\mathrm{QE}$ into NA $=0.5$ and the $\mathrm{QE}$ outside the NA (a measure of the crosstalk) for the 108-nm RCLED, the 120-nm RCLED, the highly efficient RCLED [5] and an ideal planar LED. The highly efficient RCLED has a 2-mm diameter, resulting in an increased overall $\mathrm{QE}$ due to the recycling effect. This graph shows that, by tuning the cavity, a small RCLED has the same $\mathrm{QE}$ into NA $=0.5$ at a smaller crosstalk level. The efficiency decreases at higher currents due to broader intrinsic spontaneous emission spectra and internal heating, resulting in a saturation of the optical power. The saturation power is proportional to the RCLED area, and is $1.54 \mathrm{~mW}$ for the $30-\mu \mathrm{m}$-diameter RCLED. The temperature dependence of the 


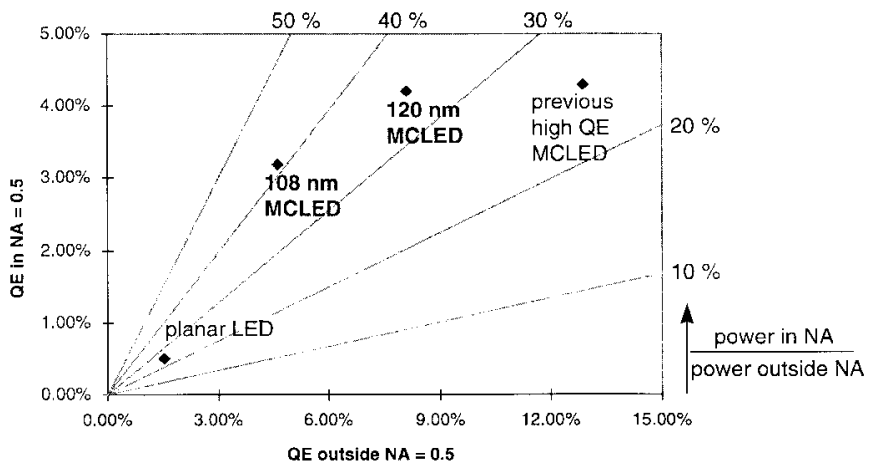

Fig. 3. Comparison of QE into NA $=0.5$ for different LED's.

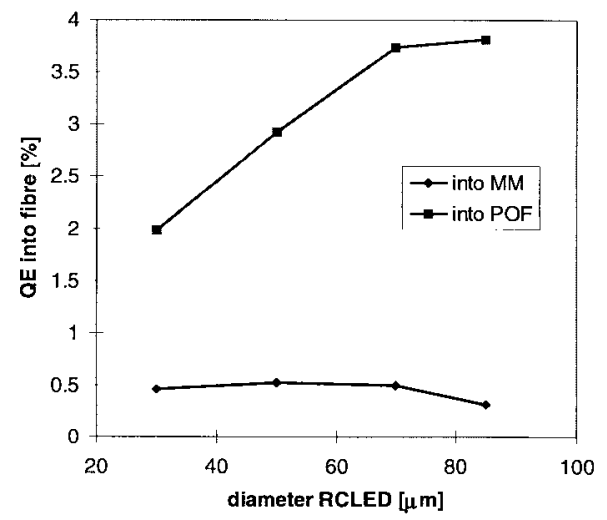

Fig. 4. Measured $\mathrm{QE}$ at 2-mA drive current into 62.5/125 multimode glass fiber and $500-\mu \mathrm{m} \mathrm{POF}$.

RCLED was evaluated, resulting in a relative power decrease of $0.8 \%$ per degree.

The coupling between the RCLED and different fiber types was evaluated. To this end, flip-chip mounted devices were aligned using an XYZ-translation block to the fibers. Two different fiber types were investigated: $62.5 / 125$ gradient index (GRIN) multimode glass fiber (MM fiber) and 500- $\mu \mathrm{m}$ POF. A difficulty in the coupling experiments with the POF's is the repeatability of the fiber facet quality. We used a hot knife technique, followed by polishing step, to obtain smooth POF surfaces [9].

The difference between the QE into NA $=0.5(4.1 \%)$ and the measured QE into the fiber (3.7\%) can be explained by reflection losses at the fiber facet and the partial overlap between the RCLED and the fiber facet. This overlap efficiency is given by the fraction of the rays emitted by the RCLED, within the acceptance angle of the fiber, that hit the fiber core. This geometrical overlap efficiency is dependent on the fiber and RCLED diameter, and the distance between RCLED and fiber. For small RCLED's close to the fiber facet, this overlap efficiency is 1 , as all light from the RCLED within the NA of the fiber hits the fiber facet. For large RCLED's positioned close to the fibers this value is proportional to $R^{-2}$ (with $R$ the radius of the RCLED active area). The reflection losses at the fiber facet includes the Fresnel losses (about 5\% at the glass-air and polymer-air interface), but also scattering due to small irregularities at the fiber facet.
Fig. 4 shows the measured QE into POF (diameter = $500 \mu \mathrm{m}$ ) and multimode glass fiber (diameter $62.5 \mu \mathrm{m}$ ) for different RCLED diameters at a constant drive current of $2 \mathrm{~mA}$. This QE obtains a maximal value for RCLED diameters roughly equal to the fiber diameter. If the RCLED diameter is smaller than the fiber diameter, the extraction efficiency decreases due to an increased current density, and larger RCLED's suffer from a low overlap efficiency. This is shown in Fig. 4, where the overall QE into MM glass fiber decreases for RCLED larger than $70 \mu \mathrm{m}$.

\section{CONCLUSION}

We fabricated planar RCLED's with an optimized cavity to maximize the extraction efficiency and minimize crosstalk. We found on a theoretical basis that there is a tradeoff between high extraction efficiency and low beam divergence. The maximum theoretical extraction efficiency into the numerical aperture of a polymer optical fiber for planar RCLED's with 30-nm intrinsic spontaneous emission spectral width is $7.8 \%$. Devices were fabricated, and the QE into NA $=0.5$ was found to be $4.1 \%$. The measured QE into $500-\mu \mathrm{m}$ POF was found to be $3.7 \%$.

\section{ACKNOWLEDGMENT}

The authors wish to acknowledge S. Verstuyft for the processing, L. Van Wassenhove for the flip-chip mounting, and A. Van Hove for supplying terminated POF.

\section{REFERENCES}

[1] G. Bjork, S. Machida, Y. Yamamoto, and K. Igeta, "Modification of spontaneous emission rate in planar dielectric microcavity structures," Phys. Rev. A, vol. 44, no. 1, pp. 669-681, July 1991.

[2] E. F. Schubert, N. E. Hunt, R. J. Malik, M. Micovic, and D. L. Miller, "Temperature and modulation characteristics of resonant-cavity lightemitting diodes," J. Lightwave Technol., vol. 14, pp. 1721-1728, July 1996.

[3] C. Wheeler, S. Daryanani, D. L. Mathine, G. N. Maracas, and D. R. Allee, "Monolithic integration of a GaAs MESFET with a resonant cavity LED using a buried oxide layer," IEEE Photon. Technol. Lett., vol. 9, pp. 194-196, Feb. 1997.

[4] D. L. Huffaker, C. C. Lin, J. Shin, and D. G. Deppe, "Resonant cavity light emitting diode with an AlxOy/GaAs reflector," Appl. Phys. Lett., vol. 66, no. 23, pp. 3096-3099, June 1995.

[5] H. De Neve, J. Blondelle, P. Van Daele, P. Demeester, R. Baets, and G. Borghs, "Recycling of guided mode light emission in planar microcavity light emitting diodes," Appl. Phys. Lett., vol. 70, no. 7, pp. 799-801, Feb. 1997.

[6] H. De Neve, J. Blondelle, R. Baets, P. Demeester, P. Van Daele, and G. Borghs, "High efficiency planar microcavity LED's: Comparison of design and experiment," IEEE Photon. Technol. Lett., vol 7, pp. 287-289, Mar. 1995.

[7] R. Bockstaele, A. Van Hove, T. Coosemans, C. Sys, I. Moerman, B. Dhoedt, R. Baets, P. Van Daele, J. Van Koetsem, and L. Van der Torren, "Micocavity LED based parallel data link using small diameter $(125 \mu \mathrm{m})$ plastic optical fibers," in Proc. SPIE Optics in Computing'98, Brugge, Belgium, vol. 3490, pp. 293-296.

[8] K. Harada, "LED array modules by new technology microbump bonding method," IEEE Trans. Comp., Hybrids, Manufact. Technol., vol 13, pp. 521-527, 1990

[9] A. Van Hove, T. Coosemans, B. Dhoedt, P. Van Daele, and R. Baets "Termination of small diameter (125 micrometer) plastic optical fiber for $1 \times 12$ datacommunication," in Proc. ECTC'98, Seattle, WA, pp. $783-798$. 